\title{
Reconfiguración del aula escolar - un análisis sensorio de la interacción alumno-maestro
}

\author{
Reconfiguration of the classroom environment - A sensorial analysis of the teacher - pupil \\ interaction
}

\author{
Cardellino, Paula \\ Universidad ORT Uruguay, Uruguay \\ cardellino@ort.edu.uy
}

\author{
Araneda, Claudio \\ Universidad del Bío-Bío, Chile \\ claraneda73@gmail.com
}

\begin{abstract}
This paper presents a strategy for experiential analysis in the traditional classroom environment to determine architectural features to contribute to the understanding and improvement of the pupil - teacher interaction. The methodology includes the analysis of the following topical propositions: visual, proxemic and kinetic in four classroom environments. The conclusions suggest that this hybrid methodological corpus allows for the identification and quantification of significant perceptual variations in relation to the proportion of the classroom space. It also emphasizes the importance of integrating aspects of sensory based analysis in the architectural design process to ensure quality of relations in the classroom.
\end{abstract}

Keywords: aula escolar; interacción maestra-alumno; diseño arquitectónico; análisis vivencial.

\section{Introducción}

El proceso arquitectónico implica el diseño de espacios capaces de acoger distintas actividades. Sin embargo, en el ámbito del diseño educativo, por lo general, no se utilizan procedimientos formales de análisis de la ocupación y comportamiento de los usuarios en el espacio. Esto es particularmente notable en el aula escolar donde su forma, tamaño y dimensiones han permanecido inmutables a pesar de las sugerencias de las nuevas tendencias educativas sobre la necesidad de distintas configuraciones espaciales. Una uniformidad formal basada en condiciones constructivas con escasas referencias a cambios sociales y particularidades funcionales.

La utilización de metodologías de registro y estrategias de análisis del tipo vivencial, que han demostrado aportes en diversas áreas del conocimiento, permiten la identificación de condiciones del espacio escolar que contribuyan a su mejor desempeño futuro. Por tanto, la aplicación de estos medios ofrece la oportunidad de obtener una nueva perspectiva sobre la relación espacio/usuario y el espacio.

Este artículo presenta una aproximación a una metodología de análisis sensorio espacial para el análisis de la interacción maestro - alumno en el aula escolar tradicional con el objetivo de realizar un diagnóstico vivencial de dicho fenómeno. La estrategia se divide en tres relaciones que se consideran cruciales en la interacción y comunicación entre maestro y alumnos: visual, proxémica y cinética. La conclusión señala que este corpus metodológico permite precisar aspectos arquitectónicos como son la proporción, la forma, y la configuración espacial del aula escolar en función de una optimización de la relación interaccional entre maestro y alumnos. Se destaca, a su vez, la importancia de integrar el análisis vivencial de los espacios en el proceso de diseño arquitectónico para asegurar no solo su calidad, sino, además, garantizar la igualdad de condiciones de los alumnos respecto a las fuentes de información y conocimiento - pizarrón y maestra - lo que a fin de cuentas constituye un derecho humano.

\section{Análisis sensorio espacial}

El concepto de fenomenología se define como la exploración y descripción de un fenómeno, donde fenómeno refiere a los aspectos y experiencias tal y como las personas las perciben (Seamon, 2000). En este sentido, los estudios fenomenológicos prestan atención a las instancias específicas de un fenómeno con la esperanza de que estas en conjunto, en algún momento, señalen hacia cualidades y características generalizables que describan de forma más precisa su naturaleza (por ejemplo: Martin Heidegger y Maurice Merleau-Ponty). Entonces, uno de los desafíos fenomenológicos es la descripción de esta íntima relación persona-mundo de manera que legítimamente escapen de cualquier dicotomía sujeto-objeto. Como señala NorbergSchultz (1975) son las actividades de las personas en los espacios las que dan sentido a la arquitectura.

Tanto en términos filosóficos como de la experimentación, la enseñanza y la realización de la arquitectura han crecido significativamente. Las ideas de Pallasmaa (2012) sobre el papel del cuerpo humano como centro de la percepción y la conciencia, así como sobre la importancia de los sentidos en la articulación, almacenamiento y procesamiento de las respuestas y pensamientos sensoriales, se han reforzado y confirmado por numerosos escritores (Lawson, 2001; Gehl, 
2010; Holl, 2011). A pesar de la importancia que esta percepción espacial presenta para la interacción, apenas se ha introducido en el discurso teórico arquitectónico donde las teorizaciones sobre arquitectura y las prácticas proyectuales se han preocupado, principalmente, por la forma (Pallasmaa, 2012).

\section{Los sentidos y las distancias}

La relación entre arquitectura y cuerpo humano tiene una larga historia. Desde los principios de armonía y proporción de la arquitectura clásica hasta los criterios ergonómicos contemporáneos, mucho camino se ha transitado. En su tratado, Vitruvio presenta la analogía de los órdenes clásicos con los cuerpos del hombre y la mujer, aspecto que se asume durante siglos como uno de los cánones clásicos más respetados por los arquitectos del momento. La espacialidad generada por estos principios vitruvianos se relaciona con el cuerpo humano a través de las propiedades armónicas destinadas a generar orden y belleza (Saldarriaga Roa, 2002). Hoy en día, sin embargo, la relación entre la arquitectura y el cuerpo se percibe de forma diferente.

La experiencia de la arquitectura se establece a través de una base de datos perceptuales que constituyen los parámetros básicos de referencia del espacio físico. Los sentidos proveen la información sobre la materialidad del mundo y permiten construir su imagen. La forma y propiedades motrices del cuerpo humano regulan esa construcción. De la estructura simétrica del cuerpo deriva el sentido visual del equilibro en las formas. La posición de la cabeza y la altura de los ojos definen una visibilidad particular (Saldarriaga Roa, 2002). La mirada estática tiene un rango limitado de sondeo que se compensa con los movimientos rotatorios para abarcar el entorno circundante. El movimiento humano genera un espacio virtual en el que el cuerpo puede acomodarse para el reposo o el ejercicio. Los sentidos del equilibrio y de la orientación determinan los niveles básicos de la experiencia de la arquitectura.

La distancia es un aspecto significativo que tiene que ver con la relación de los sentidos con el espacio. Con independencia de los desarrollos tecnológicos y sociales, los seres humanos permanecen seres ambulantes con una altura promedio de 1,75 metros y un horizonte visual horizontal con claras limitaciones. Esta constitución básica del cuerpo, entendida como aparato sensorial del ser humano, es fundamental para entender cómo se experimenta el espacio. La distancia, por ende, no es abstracta ya que está estrechamente ligada con la forma en que las personas se relacionan entre ellas y con el entorno que las rodea. No es un simple continuo, sino que cuenta con una serie de "umbrales" de percepción que determinan la forma de interacción entre personas (Lawson, 2001).

El antropólogo americano Edward T. Hall (1959; 1969) brinda un resumen detallado de la historia evolutiva de la especie humana y una primera aproximación a la importancia de los sentidos en relación al espacio. Según Hall los sentidos pueden ser clasificados en dos grupos: los sentidos de la distancia (la vista, el oído y el olfato) y los de la cercanía (el tacto y el gusto). De entre ellos, la vista es el sentido más desarrollado. De acuerdo con Hall, a una distancia de 100 metros se puede detectar el movimiento humano y a una distancia entre 22 y 25 metros se distinguen expresiones faciales y señales emocionales. Sin embargo, la experiencia se hace más interesante cuando las personas se encuentran a menos de 7 metros de distancia ya que es allí cuando se puede comenzar a entablar una auténtica conversación y donde la interacción puede poner en juego todos los otros sentidos (Hall, 1969).

Desde los estudios seminales de Hall concernientes con el impacto que el espacio tiene sobre el comportamiento humano se sabe que el espacio hecho por el hombre influye directamente en su comportamiento así como en las relaciones humanas que dentro de él se desarrollan (Hall, 1969). Esto, a su vez, impacta en la forma en que se organiza el espacio circundante. Naturalmente, mas allá de toda prescripción, la mejor manera de testear un espacio es experimentar personalmente la relación espacial y la escala del lugar. Una vez que se comienza a medir, reunir y sistematizar las observaciones personales, los conceptos como escala humana, sentidos y necesidades toman un significado más concreto. Estos conceptos ya no son incorporados como una idea luego de finalizado el proyecto, sino se incorporan naturalmente desde el comienzo del diseño para las 'personas' (Lawson, 2001; Gehl y Svarre, 2013). Este objetivo informa y orienta esta investigación.

En la interacción entre personas la relación visual ha jugado un rol preponderante. De los doce sentidos con los que cuenta el ser humano para percibir el mundo el $80 \%$ lo realiza la vista. El ojo humano es un órgano sensorial privilegiado donde la interpretación de información resulta de las imágenes que se producen en la retina. Históricamente, en la cultura occidental, la vista ha sido considerada como el más noble de los sentidos donde la invención de la representación en perspectiva hizo del ojo el punto central del mundo perceptivo. "Las imágenes visuales registran las figuras, los colores, las texturas, las distancias, las direcciones y, en fin, todo aquello que se capta a través de la mirada" (Saldarriaga Roa, 2002). Sin embargo, la percepción visual sólo capta algunos aspectos del fenómeno. En este sentido, Saldarriaga Roa (2002) habla de la "imagen perceptual" no como algo visual sino como algo que se adquiere por medio de los demás sentidos. Gibson por su parte distingue el campo visual del mundo visual. El primero implica todo lo que se alcanza a ver, por el contrario, el mundo visual implica una selección que está determinada por aspectos sicológicos, ambientales y sociales (Gibson, 1974).

No cabe duda que la cultura tecnológica ha ordenado y separado aún con más claridad a los sentidos. Los sentidos sociales privilegiados en esta cultura no solo se remiten a la vista sino que el oído, también, se toma como primordial para la interacción. El sonido en arquitectura puede resultar completamente inadvertido, pero inconscientemente sugerente. El eco y la reverberación en los espacios, la sensación de profundidad o rigidez material se hace patente mediante el sonido. La posibilidad de escuchar a la persona, 
a su vez, es de primordial importancia para la comunicación. La distancia es un aspecto significativo que tiene que ver con la relación de los sentidos con el espacio (Hall, 1969; Sommer, 1969). El estudio del desplazamiento de las personas en un espacio determina la distancia relativa a la que se encuentran a la hora de relacionarse. Dentro de un espacio determinado el movimiento de las personas modifica las condiciones de interacción.

La interacción entre personas y de estas con el espacio involucra todos los sentidos (Gehl, 2010). Se señala también la importancia de trabajar de forma cuidadosa con los sentidos y las distancias dentro del área de la educación (Gehl, 2010; Gehl y Svarre, 2013; Lawson, 2001) donde se destacan la necesidad de un contacto visual entre maestro y alumno, además de procurar que exista una distancia adecuada entre ambos para lograr una comunicación intensa y multisensorial. A los efectos de este estudio, estas esferas comunicacionales sirven para estimar el tipo de relación de interacción que cada alumno mantiene con la maestra dentro del aula. Estas tres relaciones diferenciadas, o 'fenómenos perceptivos' como los denomina Holl (2011) son: la visual, la proxémica (relaciones humanas en relación al espacio construido) y la cinética.

\section{Proposiciones}

\section{Sobre la variable visual}

Para que sea posible profundizar en el entendimiento del espacio es crucial el estudio de la percepción espacial de las personas que es captada por los sentidos. Este conocimiento es necesario tanto para diseñar como para modificar el espacio a la escala humana (Gehl, 2010) y donde se destaca que la esfera visual juega un rol preponderante en la percepción espacial. Esta relación visual ocurre dentro del aula de forma espontánea entre alumno y maestro. En este sentido, se define como relación visual 'recomendada' cuando el alumno puede interaccionar con la fuente de información sin que ningún sentido involucrado se vea impedido de hacerlo. Sin embargo, hay condicionantes del tipo físico que tienen inferencia en esta relación.

PROPOSICIÓN 1: la cantidad de alumnos en estado de relación visual 'recomendada' con la fuente de información aumenta o disminuye según la proporción del aula y la disposición de bancas.

\section{Sobre las distancias proxémicas}

La distancia es un aspecto significativo que tiene que ver con la relación de los sentidos con el espacio ya que no es un concepto abstracto sino que está estrechamente ligado con la forma en que las personas se relacionan entre ellas y con su entorno. Las distintas formas de interacción se presentan a lo largo de las diferentes distancias, que, además, dependen del tipo de contacto que se establece y de su naturaleza.

Es dentro del aula donde estas distancias se hacen patentes; la distancia entre alumno y maestro se comienza a tornar importante cuando se hace evidente que afecta su interacción. Se considera que la distancia recomendada para la interacción es la social, según la define Hall.

PROPOSICIÓN 2: la cantidad de alumnos a distancia recomendada con el maestro está condicionada por la proporción del aula.

\section{Sobre la variable cinética}

La variable cinética está directamente relacionada con el desplazamiento de personas en el espacio. En este sentido, este estudio argumenta que existen dos grandes maneras de analizar la variable cinética. Por un lado, el desplazamiento dentro del aula se puede ver como las posibilidades que tienen los usuarios de transitar libremente por el espacio definido, no solo por la disposición espacial y las características arquitectónicas, sino también por la forma en que se lleva a cabo la clase. Es decir, la forma en que se dispone el mobiliario.

PROPOSICIÓN 3: el desplazamiento, y las posibilidades de desplazarse, de los alumnos está determinado por las características espaciales de proporción del aula y de disposición de bancas del aula.

\section{Metodología}

Aceptando desde el comienzo que todo tipo de registro de una experiencia es una reducción de la misma, la estrategia que se propone es escencialmente de corte fenoménico/inmersiva y consiste básicamente en (1) el estudio de isovistas tradicionales, (2) de isovistas fotográficas $y,(3)$ de las distancias derivadas de la proxémia.

\section{Registro visual}

En el caso del vínculo visual el registro consta de dos partes diferenciadas. Por un lado, se determinan las isovistas de carácter planimétrico abstracto bidimensional y, por otro, se efectúan isovistas vivenciales, derivadas de registros fotográficos realizados por cada alumno en dirección a las fuentes de información (pizarrón y maestra).

Las isovistas planimétricas, desarrolladas por Benedict (1979), se utilizan para estudiar espacios arquitectónicos y urbanos. Consisten en polígonos bidimensionales que describen la posible visual de un individuo del recinto 0 espacio desde una ubicación determinada. Para este estudio, las isovistas se utilizan con el objetivo de proveer de información bidimensional relativa a las 'posibilidades' visuales que cada alumno tiene desde su ubicación en el aula. En la planta arquitectónica se trazan líneas de visual que parten de la posición del alumno, como rayos, que rebotan contra los elementos que se encuentran en el campo visual del mismo. Este análisis permite constatar el potencial visual de cada alumno dentro del espacio de aula en un ángulo de 180 grados (figura 1). 
[Paper781-01.JPG ]

Figura 1: Planta de isovista bidimensional para un alumno con ángulo de 180 grados.

Por su parte, la isovista de carácter experiencial se puede describir como el campo visual 'efectivo', fotográficamente abstraído, que se obtiene desde una ubicación determinada en el espacio. Esta isovista acerca y plasma, de forma tangible y efectiva, la visual en un instante desde una ubicación determinada. Para este estudio, la isovista experiencial del alumno se obtiene a través de la captura fotográfica que el mismo realiza desde su ubicación espacial en dirección al pizarrón (figura 2). Esta imagen fotográfica proporciona datos concretos sobre los elementos que componen el campo visual del participante de donde se extraen los porcentajes de maestra, pizarrón y alumnos.

[Paper781-02.JPG ]

Figura 2: Registro fotográfico de un alumno y la distinción de porcentajes de maestra, pizarrón y alumnos.

\section{Registro de distancias proxémicas}

Estas distancias se verifican a través de las esferas de interacción que surgen de la teoría proxémica que define Hall como: íntima, personal, social y pública. Cada una de estas distancias se genera cuando dos o más personas interactúan y tienen características propias referidas al tipo de comunicación cara a cara que se lleva a cabo.

De acuerdo con Lawson (2001) dentro de la distancia social se pueden mantener conversaciones a un nivel de voz normal y es posible saber si la persona está escuchando y prestando atención y, probablemente, si está entendiendo lo dicho. Sin embargo, por fuera de esta esfera la sensación de contacto e interacción con una persona tiende a perderse. Definitivamente, más allá de la distancia social se renuncia al contacto efectivo con otras personas en un espacio determinado, pudiendo llegar a ignorarse.

Para el registro se superponen las esferas proxémicas a la planta arquitectónica del aula de acuerdo con la ubicación espacial de la maestra. A los efectos de esta investigación, se realizan tres registros distintos cuya variación se vincula con las posiciones espaciales más comunes de la maestra. De este modo, las esferas se dibujan como arcos concéntricos con centro en la maestra y plasman de forma gráfica la esfera en la cual queda ubicado el alumno. Para cada instancia de ubicación de la maestra, el alumno queda ubicado en una esfera que está determinada por la distancia que lo separa de la maestra (figura 3).

[Paper781-03.JPG ]
Figura 3: Planta de aula con distancias proxémicas correspondiente a un alumno.

\section{Registro cinético}

Para explorar la relación cinética se elige un registro de tipo vivencial en base a video donde se aplica la técnica de rastreo o tracing.

El registro del movimiento proporciona datos sobre los patrones de desplazamiento de las personas. El rastreo, entonces, implica el dibujo de líneas en un plano de acuerdo con el movimiento de las personas durante un período de tiempo determinado. Para el registro cinético dentro del aula, primeramente, se filma la clase, donde la filmadora se coloca en un lugar determinado del aula desde donde se puede captar la mayor cantidad de actividad. La filmación se realiza con el fin de registrar los desplazamientos que realizan los alumnos dentro del aula durante una hora de clase, los cuales se dibujan como líneas en la planta arquitectónica el aula (figura 4).

[Paper781-04.JPG ]

Figura 4: Registro de la relación cinética de un alumno.

\section{Discusión y conclusiones}

Esta metodología de análisis de tipo vivencial permite una aproximación al estado de la relación entre la cantidad de alumnos en óptima relación interaccional con los máximos en la proporción de profundidad del espacio de clase y la cantidad de filas en el aula.

La aplicación de esta metodología evidencia la presencia de una tensión entre las distintas proposiciones de interacción estudiadas. $Y$ esto tiene, a su vez, un efecto sobre las consideraciones de dimensión y disposición espacial del aula. El "área" utilizable de aula, en última instancia, queda demarcada por la interacción entre la maestra y los alumnos y por tanto determinada, en términos de este estudio, por el dilema que se hace evidente entre la relación visual y proxémica con la relación cinética. Parece ser que en los casos estudiados cuando se logra acomodar una relación las otras se afectan. Los resultados, entonces, indican que para lograr un acople o dialogo entre todas las proposiciones de interacción, el espacio de aula y la disposición de los alumnos debe acoger las demandas de estas condiciones por igual.

Al centrarse en las relaciones vitales que acoge el espacio, la estrategia de análisis que se plantea para el análisis del fenómeno de interacción entre alumno y maestro dentro del aula tiene el potencial de arrojar luz sobre aspectos del diseño arquitectónico fundamentales y aun así no revisados ni actualizados tales como la dimensión y proporción optimas recomendadas en favor de la interacción. Sin embargo, la contribución última de este trabajo es ampliar la comprensión y concepción del espacio arquitectónico a través del diseño y 
aplicación de una estrategia mixta de técnicas de análisis mediados y en primera persona - para el diagnóstico de aulas tradicionales, sugiriendo un corpus metodológico y una verificación de aportes en el diseño de impacto más general, que permitan abrir el camino a la mejora del diseño de la arquitectura escolar $y$, en lo posible, de la práctica profesional a traves del diseño basado en evidencia y centrado en personas.

\section{Agradecimientos}

El trabajo presentado aquí es parte de la investigación que se lleva a cabo en la Facultad de Arquitectura, Universidad ORT Uruguay en el marco del Doctorado en Arquitectura y Urbanismo que la autora realiza en la Universidad del BíoBío, Chile.

\section{Referencias}

Benedict, M. L. (1979) To take hold of space: Isovists and isovists fields. Environment and Planning B, 6, 47-65.

Gehl, J. (2010) Cities for people. Washington: Island Press.

Gehl, J. \& Svarre, B. (2013). How to study public life. Washington: Island Press.
Gibson, J.(1974). La percepción del mundo visual. Infinito.

Hall, E. (1959). The silent language. New York: Doubleday.

Hall, E. (1969). The hidden dimension. Nueva York: Anchor Books Editions.

Holl, S. (2011). Cuestiones de percepción: fenomenología de la arquitectura. Barcelona: Gustavo Gili.

Lawson, B. (2001). The language of space. Oxford: Architectural Press.

Norberg-Schultz, C. (1975). Existencia, Espacio y Arquitectura. Barcelona: Editorial Blume.

Pallasmaa, J. (2012). The eyes of the skin. Chichester: John Wiley \& Sons Ltd.

Saldarriaga Roa, A. (2002). La arquitectura como experiencia: espacio, cuerpo y sensibilidad. Bogotá: Villegas Editores.

Seamon, D. (2000). A way of seeing people and place: Phenomenology in environment-behavior research. In S.Wapner, J. Demick, T. Yamamoto \& H. Minami (Eds.), Theoretical perspectives in Environment-Behavior Research (pp. 157 - 178). New York: Plenum.

Sommer, R. (1969). Personal space. The behavioral basis of design. London: Prentice-Hall. 